\title{
Polaron transport in organic crystals: Temperature tuning of disorder effects
}

\author{
Frank Ortmann ${ }^{1,2}$ and Stephan Roche $\mathrm{e}^{2,3}$ \\ ${ }^{1}$ Commissariat à l' Energie Atomique et aux Energies Alternatives (CEA), Institut Nanosciences et Cryogénie (INAC), Structure et Propriétés \\ d'Architectures Moleculaires (SPRAM), Groupe Théorie, 17 rue des Martyrs, \\ F-38054 Grenoble Cedex 9, France \\ ${ }^{2}$ CIN2 (ICN-CSIC) and Universitat Autónoma de Barcelona, \\ Catalan Institute of Nanotechnology, Campus de la UAB, E-08193 Bellaterra (Barcelona), Spain \\ ${ }^{3}$ ICREA, Institució Catalana de Recerca i Estudis Avançats, E-08070 Barcelona, Spain
}

(Received 11 October 2011; published 18 November 2011)

\begin{abstract}
We explore polaronic quantum transport in three-dimensional models of disordered organic crystals with strong coupling between electronic and vibrational degrees of freedom. By studying the polaron dynamics in a static disorder environment, temperature-dependent mobilities are extracted and found to exhibit different fingerprints depending on the strength of the disorder potential. At low temperatures and for strong enough disorder, coherence effects induce weak localization of polarons. These effects are reduced with increasing temperature (thermal disorder), resulting in mobility increase. However, at a transition temperature, phonon-assisted contributions driven by polaron-phonon scattering prevail, provoking a downturn of the mobility. The results provide an alternative scenario to discuss controversial experimental features in molecular crystals.
\end{abstract}

DOI: 10.1103/PhysRevB.84.180302

PACS number(s): 71.38.-k, 72.80.Le, 72.15.Rn

The microscopic understanding of charge transport in organic materials remains a challenge after more than two decades of theoretical and experimental efforts., ${ }^{1,2}$ An established fact is, however, that transport theories developed for conventional inorganic semiconductors fail to properly describe organic matter (or narrow-band systems in general) in its full complexity. One of the main reasons lies in strong electron-phonon coupling which is at the basis of high technical and conceptual hurdles, demanding for the advancement of concepts and quantum transport methods that are able to cope with the entangled effects of structural or chemical and thermal disorders. For strongly disordered (and soft) organic structures (such as discotic liquid crystals, DNA, or microcrystalline polymer semiconductors), multiscale approaches combining classical molecular dynamics with quantum transport simulations offer an interesting perspective. ${ }^{3,4}$ On the other hand, since the seminal work of Su, Schrieffer, and Heeger, ${ }^{5}$ it is recognized that the electron transport in $\pi$-conjugated materials encompasses various complicated transport mechanisms, including band conduction or hopping processes which can be well captured by the concept of polarons.

After early works by Holstein ${ }^{6}$ and Conwell, ${ }^{7}$ theoretical progress in recent years has been achieved in the exploration of polaronic phenomena, including the description of Fröhlich polarons at the dielectric/organics interface in organic-field-effect transistors (accounting for Coulomb interaction and screening phenomena), ${ }^{8}$ as well as the simulation of nonadiabatic polaron motion, ${ }^{9}$ inelastic effects on coherent polaronlike motion, ${ }^{10}$ or the revision of dynamic localization concepts in one-dimensional models of organic materials. ${ }^{11}$ Additionally, to investigate polaron motion in higher dimensional [three-dimensional (3D)] systems, a generalized transport methodology has been proposed using a mixed Holstein-Peierls model ${ }^{12}$ in the Kubo framework. This latter work has provided some qualitative understanding of high-temperature-dependent charge mobility of ultrapure organic crystals (including anisotropic effects), but it does not explain the sign reversal of the bulk mobility versus temperature derivative $\left(\frac{\partial \mu}{\partial T}\right)$ in the lower-temperature regime, ${ }^{13}$ which is still a debated issue. Indeed, as observed by Karl and co-workers in $\alpha$-perylene single-crystalline thin films, charge mobilities can increase from $1 \mathrm{~cm}^{2}(\mathrm{~V} \mathrm{~s})^{-1}(10 \mathrm{~K})$ to $\sim 100 \mathrm{~cm}^{2}(\mathrm{~V} \mathrm{~s})^{-1}$ at $30 \mathrm{~K}$, followed by a power-law decay up to room temperature. ${ }^{13} \mathrm{~A}$ similar mobility behavior also has been more recently reported in rubrene crystal-based field-effect transistors ${ }^{14}$ and in modified pentacene polycrystalline films with large domain sizes of over $100 \mathrm{~nm} .{ }^{15}$ Notwithstanding repeated analysis, the underlying physical mechanism driving such temperature-dependent mobility remains elusive. ${ }^{16}$ Moreover, the reported clear evidences for band dispersion (as large as $400 \mathrm{meV}$ ) in high-purity rubrene single crystals ${ }^{17}$ support the scenario of polaron band transport, ${ }^{11,18,19}$ although the question of quantum interference effects ${ }^{20}$ remains to be investigated and quantified in 3D disordered organic crystals.

In this Rapid Communication, we use a real-space order $N$ Kubo methodology to theoretically explore polaron transport in three-dimensional disordered organic crystals, taking into account elastic scattering, as well as decoherence effects. Decoherence is introduced at a phenomenological level, assuming that the coherent polaron motion suffers from additional stochastic dephasing events, driven by thermal vibrations which play an important role in the low- $T$ regime. ${ }^{21}$ Additionally, incoherent contributions of polaron motion (driven by polaron-phonon scattering ${ }^{12}$ ) are found to dominate the high- $T$ regime. The obtained temperature-dependent mobility fingerprints are reminiscent of experimental features in fairly ordered organic materials. ${ }^{13-15}$

The present theoretical approach uses a polaronic tightbinding (TB) Hamiltonian and orthorhombic symmetry (see the inset of Fig. 1), as found in rubrene, which is defined by $H=H_{P}+H_{W}$, where $H_{P}=\sum_{m \neq n} \widetilde{\varepsilon}_{m n} a_{m}^{\dagger} a_{n}+\sum_{\lambda} \hbar \omega_{\lambda} b_{\lambda}^{\dagger} b_{\lambda}$ describes polarons and phonons. It can be obtained from a polaronic transformation (Lang-Firsov) ${ }^{12}$ of a generic model for disordered polaronic materials. ${ }^{22}$ Here, we use the concept 

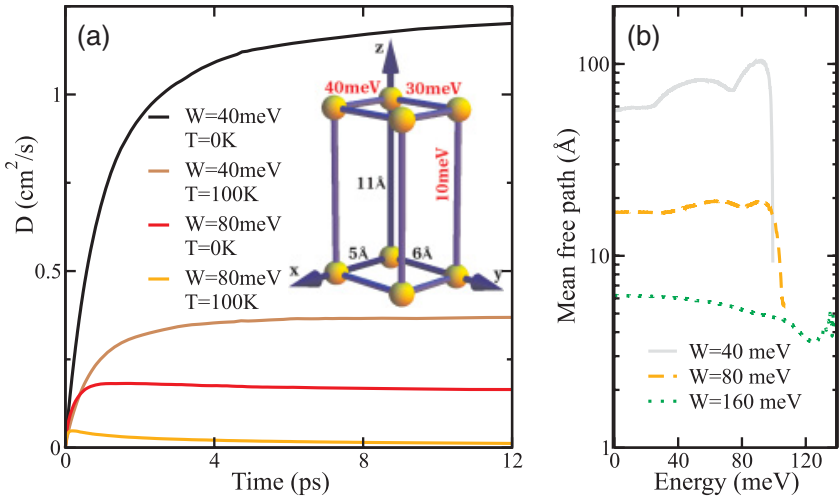

FIG. 1. (Color online) (a) Typical time-dependent diffusion coefficient for various $W$ and temperature $T$. Inset: 3D crystal structure with system parameters $\varepsilon_{m n}$ and $R_{m n}$ for nearest neighbors. (b) Polaron mean free path vs energy for various disorder strengths. Only positive energies are shown because of symmetry.

of a single effective vibrational mode with energy $\omega_{\lambda}=$ $12 \mathrm{meV}$, and we use as a typical dimensionless electronphonon coupling $g_{\lambda}=0.7$ inspired by available $a b$ initio data. The effective mode used here is representative of the many low-frequency intermolecular modes which are present in organic materials. The temperature-dependent renormalized transfer integrals $\widetilde{\varepsilon}_{m n}$ are used following the full dressing approximation $^{12}\left(N_{\lambda}\right.$ is the Bose-Einstein function), and are given by $\widetilde{\varepsilon}_{m n}=\varepsilon_{m n} \exp \left[-\sum_{\lambda}\left(1+2 N_{\lambda}\right) g_{\lambda}^{2}\right]$, with $\varepsilon_{m n}$ the electronic transfer integrals (given in the inset of Fig. 1). $H_{P}$ gives rise to a gapless cosine-type temperature-dependent and anisotropic band structure in the 3D Brillouin zone, regarded as a typical case for organic crystals. ${ }^{23}$ Finally, $H_{W}=\sum_{m} \varepsilon_{m} a_{m}^{\dagger} a_{m}$ tunes the strength of an additional elastic (Anderson-type) disorder potential ${ }^{18}$ with on-site energies taken at random within $\varepsilon_{m} \in[-W / 2, W / 2]$, with an average modulus of $\varepsilon_{m}$, which is $W / 4$. Such uncorrelated disorder mimics impurities and defects as a first approximation. ${ }^{18} \mathrm{~A}$ refined analysis could be achieved by using first-principles calculations and precise crystal symmetry (see, e.g., Ref. 24 for details).

The TB model is implemented into a Kubo transport framework which is based on the theory developed in Ref. 25. This theory is generalized in the present Rapid Communication to investigate effects of static disorder. Due to the splitting of the mobility into two contributions (coherent and incoherent) obtained in Ref. 25, we proceed here by employing two complementary coherent and incoherent Kubo transport methodologies to explore the whole temperature range. The first one allows a real-space description of polaron propagation in the coherent regime in the superimposed disordered (static) potential by using the Kubo-Greenwood approach. ${ }^{26}$ By following the polaron dynamics, the elastic polaron mean free path as well as the weak-localization corrections to the conductivity in the low-temperature regime can be computed. Second, the incoherent (phonon-assisted) contribution of polaron transport is conveyed by polaron-phonon scattering. It is calculated based on the theory developed in Ref. 25, which is here generalized to include static disorder effects (details below). Both contributions (coherent and incoherent) are computed separately and are added to evaluate the total polaron mobility. We note that there is no further parameter to tune the relative amplitudes of both. They are fixed by material parameters and the impact of disorder.

From the study of the coherent polaron wave-packet dynamics, the elastic mean free path $\ell_{e}(E)$ can be first derived from the time dependence of the diffusion coefficient $D(E, t)$, which writes

$$
\frac{1}{t} \frac{\sum_{l}\left\langle\Psi_{l}\left|\delta(E-H)\left[U^{\dagger}(t) x U(t)-x\right]^{2}\right| \Psi_{l}\right\rangle}{\sum_{l}\left\langle\Psi_{l}|\delta(E-H)| \Psi_{l}\right\rangle},
$$

taking $x$ as the position operator along the transport direction, $\delta(E-H)$ the spectral measure operator the trace of which gives the total density of states, and $\left|\Psi_{l}\right\rangle$ a set of randomphase states (for details, see Ref. 26). In this approach $D(t)$ is derived from to the time evolution of $\left\langle[x(t)-x(0)]^{2}\right\rangle$ driven by the operator $\hat{U}(t)=\Pi_{n=1}^{N_{t}} \exp (i H \Delta t / \hbar)$, with $\Delta t$ the chosen time step. The calculations are performed for total elapsed computational times of $t_{\max }=61 \mathrm{ps}$. The system size is at least $(0.24 \times 0.22 \times 0.14) \mu \mathrm{m}^{3}$ and periodic boundary conditions are applied. Based on this, the coherent part of the Kubo conductivity is computed using $\sigma\left(E, t=N_{t} \Delta t\right)=$ $e_{0}^{2} \rho(E) D(E, t) / 2$, with $\rho(E)$ the total density of states. We further derive the carrier mobility

$$
\mu^{(\mathrm{coh})}=\frac{\Omega}{e_{0} c k_{B} T} \int d E \sigma(E, t) f_{\mathrm{FD}}(E)\left[1-f_{\mathrm{FD}}(E)\right],
$$

with $f_{\mathrm{FD}}(E)$ being the Fermi-Dirac function, $c$ the total charge density fixed to $10^{-3}$, and $\Omega$ the volume of the unit cell. The chosen time $t$ is fixed by the temperature-dependent decoherence time $\tau_{\varphi}$.

By introducing static disorder, polaron wave packets are elastically scattered and suffer from multiple scattering events and quantum interferences. Elastic scattering first results in a diffusive regime, characterized by the polaron elastic mean free path $\ell_{e}=D^{\max } / 2 \widetilde{v}$ ( $D^{\max }$ is the maximum diffusion coefficient and $\widetilde{v}$ the polaron velocity). Figure 1(b) shows $\ell_{e}$ along the transport direction, which follows an approximate $\ell_{e} \propto W^{-2}$ behavior for small $W$ (in agreement with the Fermi golden rule). The obtained values for $\ell_{e}$ are well below the total system size. When $\ell_{e}$ becomes close to the lattice constant, it, however, ceases to be a suitable characteristic length as the system proceeds to the strong localization regime. In that limit the localization length $\xi$ might be used to further characterize localized states. As seen in Fig. 1(b), the case of $W=160 \mathrm{meV}$ may be regarded (in our model) as a transition point. One also notes that the polaron bandwidth varies with temperature (renormalization of transfer integrals). As a result, for a given static disorder potential, backscattering efficiency will increase with temperature.

Beyond the diffusive regime, quantum interference effects (QIEs) produce an increase of the resistance (weak localization). Strong (coherent) localization effects due to disorder in organic crystals have been studied in one- and two-dimensional models ${ }^{18}$ for which all states are localized irrespective to the disorder strength. In three-dimensional models, the validity of band-type conduction is known to be more robust with the existence of a metal-insulator transition, which can be tuned by the strength of elastic disorder. ${ }^{20}$ 
In general, quantum coherence (and localization effects) will be reduced by decoherence mechanisms. ${ }^{20}$ Decoherence mechanisms for polaron transport could be driven by thermal disorders including phonon-phonon, electron-electron, as well as electron-phonon scattering processes. This thermal disorder includes stochastic scattering events which relate the selected coherent polaron with other phonon degrees of freedom. We introduce phenomenologically a coherence time which tunes the weak localization correction, and decreases with temperature following a power law. Although there is no available microscopic theory of decoherence in inorganic (or organic) materials, a power-law dependence of the decoherence time $\tau_{\varphi} \propto 1 / T^{\alpha}$ has been derived, with $\alpha$ only depending on the transport dimensionality for electron-electron interaction induced dephasing ${ }^{20}$ (for temperatures below $1 \mathrm{~K}$ ), in contrast with the case of electron-phonon driven decoherence effects for which $\alpha \simeq 2-4$ (material dependent). ${ }^{21}$ Here we assume such a power law for $\tau_{\varphi}$ to be generic, but we take into account some variability in the $T$-dependent tuning of QIEs by considering different values for $\alpha$. We also assume that, at $5 \mathrm{~K}, \tau_{\varphi}$ corresponds to the maximum computed time $\left(\tau_{\varphi}=t_{\max }\right)$ and introduce a lower limit for $\tau_{\varphi}$ which ensures a seamless transition to the semiclassical limit at elevated $T$.

Figure 2 shows $\mu^{(\mathrm{coh})}(T)$ for various disorder strengths $W$. For values as low as $40 \mathrm{meV}$ [corresponding to elastic mean free paths in the order of 6-10 nm, Fig. 1(b)], no weak localization is observed within the reach of our maximum computed time. This can be appreciated by the absence of a time-dependent decay of the diffusion coefficient [see Fig. 1(a)]. As a result, the low-temperature $\mu^{(\mathrm{coh})}(T)$ is seen to saturate to its semiclassical value on the order of $200 \mathrm{~cm}^{2}(\mathrm{~V} \mathrm{~s})^{-1}$ for the chosen parameters. In contrast, for a static disorder strength such as $W=80$ and $160 \mathrm{meV}$ (corresponding to $\ell_{e} \sim 2 \mathrm{~nm}$ and $6 \AA$, respectively) substantial contributions of QIEs are obtained, as witnessed by the timedependent decay of the diffusion coefficient [Fig. 1(a)], and result in a marked decrease of $\mu^{\text {(coh) }}$ at the lowest temperatures. By increasing $W$ by a factor of 2 (or 4$), \mu^{(\operatorname{coh})}(T)$ is reduced

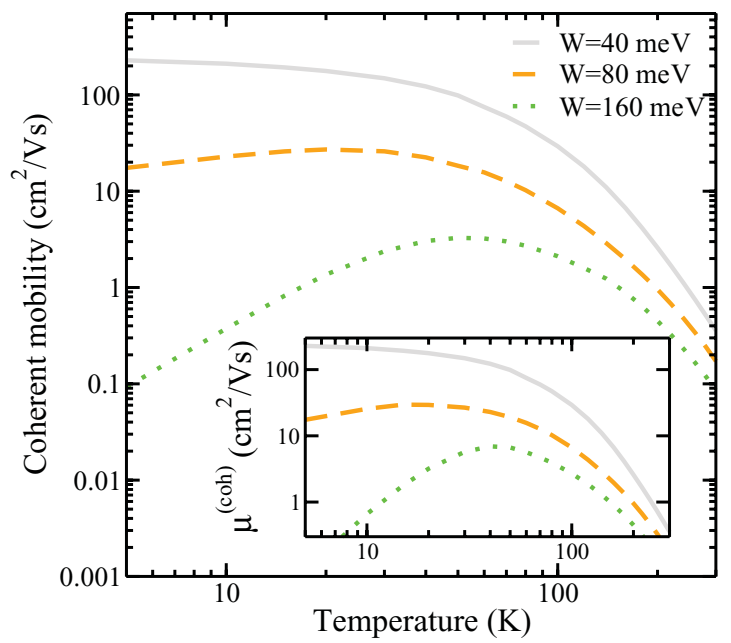

FIG. 2. (Color online) Coherent part of the carrier mobility for several disorder strengths $W$ and using a decoherence model with $\alpha=2$ (see text). Inset: Same information using $\alpha=3$. by one (or three) orders of magnitude. The temperaturedependent decoherence effects then yield a positive $\frac{\partial \mu}{\partial T}$ (Fig. 2) which is independent of the exact details of the decoherence strength introduced phenomenologically. Such a behavior can be observed only if the temperature-induced reduction of the QIE dominates the band narrowing effect, which is not observed for the negative $\frac{\partial \mu}{\partial T}$ found at higher temperatures in Fig. 2. Importantly, in contrast to the common belief that a mobility increase with temperature implies a phonon-assisted transport regime, we obtain a positive $\frac{\partial \mu}{\partial T}$ for coherent motion. This behavior can be expected to be relatively insensitive to the strength of the electron-phonon coupling or the efficiency of the phonon dressing of carriers assumed in the polaron Hamiltonian.

At elevated temperatures, the phonon-assisted incoherent contribution of polaron transport (driven by phonon-polaron scattering) may prevail over the coherent one. By generalizing Eq. (43) of Ref. 25 [using the density of states $\rho(E)$ of the disordered system], we compute the incoherent part of the polaron mobility $\mu^{(\text {inc) }}=\frac{e_{0} \Omega^{2}}{2 c \hbar^{2} k_{B} T} \sum_{m}$ $R_{m n}^{2} \widetilde{\varepsilon}_{m n}^{2} \int d E_{1} d E_{2} \rho\left(E_{1}\right) \rho\left(E_{2}\right) f_{\mathrm{FD}}\left(E_{1}\right)\left[1-f_{\mathrm{FD}}\left(E_{2}\right)\right] \int_{-\infty}^{\infty} d t$ $e^{i t\left(E_{1}-E_{2}\right) / \hbar}\left\{\exp \left[2 \Phi_{\lambda}(t) g_{\lambda}^{2}\right]-1\right\}$ in the transport direction with $\Phi_{\lambda}(t)=N_{\lambda} e^{i \omega_{\lambda} t}+\left(1+N_{\lambda}\right) e^{-i \omega_{\lambda} t}$ and display its temperature dependence in Fig. 3. ${ }^{27}$ We observe an activation behavior of the phonon-assisted transport which is followed by a saturation regime at $\sim 200 \mathrm{~K}$ and a weak decrease up to room temperature. Although reminiscent of the experimental data reported recently, ${ }^{13-15}$ we discuss below why phonon-assisted transport is unlikely to explain the mobility downturn at low $T$. We plot in Fig. 3 (main frame) the total carrier mobility $\mu^{\text {(coh) }}(T)+\mu^{\text {(inc) }}(T)$, which is the measurable quantity in experiments. For all temperatures one observes a disorder dependence of the mobility which is weaker for the high- $T$ regime. However, its origin in this regime is very different from the strong quantum interferences described in Fig. 2 at low $T$. Importantly, we find that even for strong disorder the weak localization regime of coherent motion dominates the low-temperature regime of the total carrier mobility, resulting

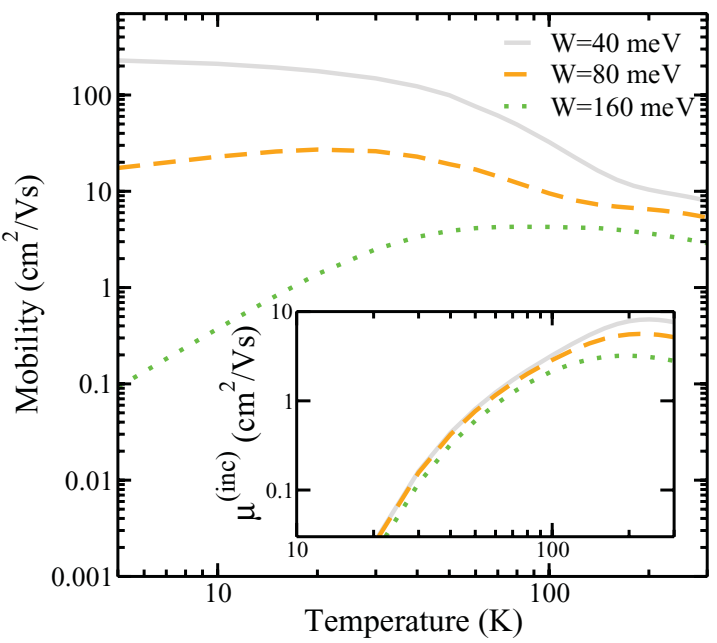

FIG. 3. (Color online) Temperature dependence of the total mobility (main frame) and incoherent part (inset) for several disorder strengths $W$ and decoherence effects with $\alpha=2$. 
in $\frac{\partial \mu}{\partial T}>0$ for strong enough disorder (not phonon assisted). On the other hand, the mobility decrease $\left(\frac{\partial \mu}{\partial T}<0\right)$ at elevated $T$ is mostly driven by phonon-assisted polaron motion, i.e., increasing contribution of carrier-phonon scattering, in contrast to the temperature-activation concept commonly associated with phonon-assisted transport.

We have studied a quantum-based polaron transport scenario and found that quantum interferences can play an important role for understanding temperature-dependent transport features. Such a scenario is consistent with experimental trends reported in clean organic crystals, ${ }^{13-15}$ and the obtained results are not within the reach of restricted semiclassical transport approaches. Although the absolute values of charge mobilities are comparable to what is measured experimentally, a systematic comparison with experimental data, including transport anisotropy, would demand the use of first-principles calculations for the material parameters.

This work is supported by the European Community through the Marie Curie Actions.
${ }^{1}$ M. E. Gershenson, V. Podzorov, and A. F. Morpurgo, Rev. Mod. Phys. 78, 973 (2006); D. Braga and G. Horowitz, Adv. Mater. 21, 1473 (2009).

${ }^{2}$ J.-L. Brédas, J. P. Calbert, D. A. da Silva Filho, J. Cornil, Proc. Natl. Acad. Sci. USA 99, 5804 (2002); A. M. Stoneham, M. M. D. Ramos, A. M. Almeida, H. M. G. Correia, R. M. Ribeiro, H. Ness, and A. J. Fisher, J. Phys. Condens. Matter 14, 9877 (2002); D. L. Cheung and A. Troisi, Phys. Chem. Chem. Phys. 10, 5941 (2008); S. Stafström, Chem. Soc. Rev. 39, 2484 (2010); V. Coropceanu, J. Cornil, D. A. da Silva Filho, Y. Olivier, R. Silbey, and J.-L. Brédas, Chem. Rev. 107, 926 (2007).

${ }^{3}$ A. Troisi and G. Orlandi, Phys. Rev. Lett. 96, 086601 (2006); A. Troisi, D. L. Cheung, and D. Andrienko, ibid. 102, 116602 (2009); D. L. Cheung, D. P. McMahon, and A. Troisi, J. Am. Chem. Soc. 131, 11179 (2009); L. J. Wang, Q. K. Li, Z. G. Shuai, L. P. Chen, and Q. Shi, Phys. Chem. Chem. Phys. 12, 3309 (2010).

${ }^{4}$ R. Gutiérrez, R. A. Caetano, B. P. Woiczikowski, T. Kubar, M. Elstner, and G. Cuniberti, Phys. Rev. Lett. 102, 208102 (2009).

${ }^{5}$ A. J. Heeger, S. Kivelson, J. R. Schrieffer, and W. P. Su, Rev. Mod. Phys. 60, 781 (1988).

${ }^{6}$ T. Holstein, Ann. Phys. 8, 343 (1959).

${ }^{7}$ E. M. Conwell, Phys. Rev. B 22, 1761 (1980).

${ }^{8}$ I. N. Hulea, S. Fratini, H. Xie, C. L. Mulder, N. N. Iossad, G. Rastelli, S. Ciuchi, and A. F. Morpurgo, Nat. Mater. 5, 982 (2006); S. Ciuchi and S. Fratini, Phys. Rev. B 79, 035113 (2009); S. Fratini and S. Ciuchi, Phys. Rev. Lett. 103, 266601 (2009); S. J. Konezny, M. N. Bussac, and L. Zuppiroli, Phys. Rev. B 81, 045113 (2010); N. G. Martinelli, M. Savini, L. Muccioli, Y. Olivier, F. Castet, C. Zannoni, D. Beljonne, and J. Cornil, Adv. Funct. Mater. 19, 3254 (2009).

${ }^{9}$ A. A. Johansson and S. Stafström, Phys. Rev. B 69, 235205 (2004).

${ }^{10}$ H. Ness, S. A. Shevlin, and A. J. Fisher, Phys. Rev. B 63, 125422 (2001); H. Ness and A. J. Fisher, Phys. Rev. Lett. 83, 452 (1999); Proc. Natl. Acad. Sci. USA 102, 8826 (2005).

${ }^{11}$ A. Troisi, Phys. Rev. B 82, 245202 (2010).

${ }^{12}$ K. Hannewald and P. A. Bobbert, Phys. Rev. B 69, 075212 (2004); K. Hannewald, V. M. Stojanovic, and P. A. Bobbert, J. Phys. Condens. Matter 16, 2023 (2004); K. Hannewald and P. A. Bobbert, Appl. Phys. Lett. 85, 1535 (2004).
${ }^{13}$ N. Karl, K.-H. Kraft, J. Marktanner, M. Münch, F. Schatz, R. Stehle, and H.-M. Uhde, J. Vac. Sci. Technol. A 17, 2318 (1999).

${ }^{14}$ V. Podzorov, E. Menard, A. Borissov, V. Kiryukhin, J. A. Rogers, and M. E. Gershenson, Phys. Rev. Lett. 93, 086602 (2004).

${ }^{15}$ T. Sakanoue and H. Sirringhaus, Nat. Mater. 9, 736 (2010).

${ }^{16}$ Z. Q. Ren, L. E. McNeil, S. Liu, and C. Kloc, Phys. Rev. B 80, 245211 (2009); A. Girlando, L. Grisanti, M. Masino, I. Bilotti, A. Brillante, R. G. Della Valle, and E. Venuti, ibid. 82, 035208 (2010).

${ }^{17}$ S. I. Machida, Y. Nakayama, S. Duhm, Q. Xin, A. Funakoshi, N. Ogawa, S. Kera, N. Ueno, and H. Ishii, Phys. Rev. Lett. 104, 156401 (2010).

${ }^{18}$ M. Unge and S. Stafström, Phys. Rev. B 74, 235403 (2006); J. D. Picon, M. N. Bussac, and L. Zuppiroli, ibid. 75, 235106 (2007); A. Troisi, J. Chem. Phys. 134, 034702 (2011).

${ }^{19}$ F. Ortmann, F. Bechstedt, and K. Hannewald, New J. Phys. 12, 023011 (2010).

${ }^{20}$ E. Akkermans and G. Montambaux, in Mesoscopic Physics of Electrons and Photons (Cambridge University Press, Cambridge, UK, 2007).

${ }^{21}$ S. Chakraverty and A. Schmid, Phys. Rep. 140, 193 (1986); J. J. Lin and J. P. Bird, J. Phys. Condens. Matter 14, R501 (2002).

${ }^{22}$ F. X. Bronold, A. Alvermann, and H. Fehske, Philos. Mag. 84, 673 (2004).

${ }^{23}$ R. C. Hatch, D. L. Huber, and H. Höchst, Phys. Rev. Lett. 104, 047601 (2010).

${ }^{24}$ V. Stehr, J. Pfister, R. F. Fink, B. Engels, and C. Deibel, Phys. Rev. B 83, 155208 (2011); Z. Shuai, L. Wang, and Q. Li, Adv. Mater. 23, 1145 (2011); F. Ortmann, K. Hannewald, and F. Bechstedt, J. Phys. Chem. B 113, 7367 (2009)

${ }^{25}$ F. Ortmann, F. Bechstedt, and K. Hannewald, Phys. Rev. B 79, 235206 (2009).

${ }^{26}$ S. Roche and D. Mayou, Phys. Rev. Lett. 79, 2518 (1997); H. Ishii, F. Triozon, N. Kobayashi, K. Hirose, and S. Roche, C. R. Phys. 10, 283 (2009).

${ }^{27}$ A phenomenological parameter $\tau$ which fixes the absolute mobility values in Ref. 25 is no longer necessary and omitted here. 